ISSN 2179-6750

\title{
Saberes e práticas de médicos e enfermeiros relativos ao pré-natal odontológico
}

\author{
Knowledge and practices of doctors and nurses relative to \\ Dental prenatal care
}

\section{Conocimiento y prácticas de los médicos y enfermeras en relación con la atención prenatal odontológica}

\author{
Rejane Marques PEREIRA ${ }^{(1)}$ \\ Suélem Maria Santana PINHEIRO-FERREIRA ${ }^{(2)}$ \\ Rogério Vieira SILVA ${ }^{(1)}$ \\ Jinária Fernandes da SILVA ${ }^{(1)}$ \\ Isis Cardoso Benício dos SANTOS ${ }^{(1)}$
}

Recebido: 11 dez 2017

Revisado: 30 maio 2018

Aceito: 27 jun 2019

Autor de

correspondência:

Suélem Maria Santana

Pinheiro Ferreira

suelem.pinheiro@gmail.com

Conflito de interesses:

Os autores declaram não

haver nenhum interesse

profissional ou pessoal que

possa gerar conflito de

interesses em relação a este manuscrito.

\footnotetext{
${ }^{(1)}$ Faculdade Independente do Nordeste - FAINOR, Departamento de Odontologia, Vitória da Conquista, BA, Brasil.

${ }^{(2)}$ Universidade Estadual de Feira de Santana, UEFS, Departamento de Saúde, Feira de Santana, BA, Brasil.
}

\section{Resumo}

O pré-natal odontológico visa identificar riscos à saúde bucal, necessidade de tratamento odontológico e promover a educação para a saúde materno-infantil. Objetivou-se descrever conhecimentos e práticas de médicos e enfermeiros envolvidos no cuidado pré-natal referentes à saúde bucal de gestantes na atenção básica de saúde de Vitória da Conquista, BA, em 2015. Trata-se de um estudo transversal, censitário e descritivo realizado com médicos e enfermeiros da atenção básica. Aplicou-se questionário objetivo para investigação socioeconômica, dos conhecimentos e ações em saúde bucal no prénatal. Os dados foram analisados de forma descritiva com frequências absolutas e relativas, e medidas de tendência central e dispersão, através do SPSS. Dos 54 entrevistados, 68,5\% estão inseridos em Unidade de Saúde da Família, com atuação média de 18 meses; 68,5\% são enfermeiros. Apenas 38,9\% participaram do curso introdutório da atenção básica, mas sem abordagem sobre a saúde bucal de gestantes $(66,7 \%)$. A maioria $(96,3 \%)$ considerou o contato da gestante com o cirurgião-dentista importante independente de queixa bucal; $75,5 \%$ incentivam as gestantes a procurarem atendimento odontológico, porém apenas $20,4 \%$ realizam inspeção visual da cavidade oral regularmente. As discussões com o Cirurgião-Dentista são raras (25,9\%) ou ausentes (31,5\%), e alguns mitos ainda persistem entre os profissionais, como a contraindicação de radiografia $(57,7 \%)$ e procedimentos cirúrgicos para gestantes $(35,2 \%)$. Conclui-se que os profissionais demonstraram maior conhecimento sobre questões gerais do pré-natal odontológico, em detrimento de aspectos norteadores de práticas. Além disso, houve baixa participação no cuidado compartilhado, com transferência da responsabilidade sobre a saúde bucal exclusivamente para o dentista.

Descritores: Conhecimentos, Atitudes e Prática em Saúde; Cuidado Pré-Natal, Saúde Bucal. 


\begin{abstract}
The oral prenatal care aims to identify risks to oral health, the need of dental treatment and to promote education for maternal and child health. The objective of this study was to describe the knowledge and practices of doctors and nurses involved in prenatal care related to the oral health of pregnant women in primary health care in Vitória da Conquista, Bahia, in 2015. This is a crosssectional, census and descriptive study conducted with primary care physicians and nurses. An objective questionnaire was apply to socio-economic research, knowledge and actions on oral health in prenatal care. The data were analyzed descriptively with absolute and relative frequencies and measures of central tendency and dispersion through SPSS. Of the 54 interviewees, $68.5 \%$ are inserted in the Family Health Unit, with an average of 18 months; $68.5 \%$ are nurses. Only $38.9 \%$ participated in the introductory course of primary care, but no approach on the oral health of pregnant women (66.7\%). The majority (96.3\%) considered the contact of the pregnant woman with the dentist is important independent of oral complaint; $75.5 \%$ encourage pregnant women to seek dental care, but only $20.4 \%$ perform visual inspection of the oral cavity regularly. Discussions with the Dental Surgeon are rare $(25.9 \%)$ or absent $(31.5 \%)$, and some myths still persist among professionals, such as radiographic contraindications (57.7\%) and surgical procedures for pregnant women (35.2\%). Concludes that the professionals demonstrated greater knowledge about general prenatal dental issues, to the detriment of guiding aspects of practices. Besides that, there was low participation in shared care, with transfer of responsibility on oral health exclusively to the dentist.
\end{abstract}

Keywords: Health Knowledge, Attitudes, Practice; Prenatal Care; Oral Health.

\begin{abstract}
Resumen
El prenatal odontológico busca identificar riesgos a la salud bucal (SB), necesidad de tratamiento odontológico y promover la educación para la salud materno-infantil. Se objetivó describir conocimientos y prácticas de profesionales involucrados en el cuidado prenatal sobre la salud bucal de gestantes en la Atención Básica de Vitória da Conquista-BA en 2015. Es un estudio transversal, censal y descriptivo con médicos y enfermeros de la atención básica. Se aplicó cuestionario para investigación socioeconómica, conocimientos y acciones en SB en el prenatal. Los datos fueron analizados de forma descriptiva con frecuencias absolutas y relativas, medidas de tendencia central y dispersión, a través del SPSS. De los 54 entrevistados, 68,5\% están insertos en Unidad de Salud de la Familia, con actuación promedio de 18 meses; el 68,5\% son enfermeros. Sólo el 38,9\% participó del curso introductorio de la atención básica, pero sin abordaje sobre la SB de gestantes $(66,7 \%)$. La mayoría $(96,3 \%)$ consideró el contacto de la gestante con el Cirujano-Dentista importante independiente de queja bucal; $75,5 \%$ incentivan a las gestantes a buscar atención odontológica, pero sólo el 20,4\% realizan la inspección visual de la cavidad oral regularmente. Las discusiones con el Cirujano-Dentista son raras $(25,9 \%)$ o ausentes $(31,5 \%)$, y algunos mitos persisten entre los profesionales, como la contraindicación de radiografía $(57,7 \%)$ y procedimientos quirúrgicos $(35,2 \%)$. Los profesionales demostraron mayor conocimiento sobre cuestiones generales del PNO, en detrimento de aspectos prácticos. Además, hubo baja participación en el cuidado compartido, con transferencia de la responsabilidad sobre la SB exclusivamente para el dentista.
\end{abstract}

Palabras-claves: Conocimientos, Actitudes y Práctica en Salud; Atención Prenatal; Salud Bucal.

\title{
Introdução
}

Os princípios que norteiam o funcionamento do Sistema Único de Saúde - SUS no Brasil contemplam, entre outros, a integralidade como forma de cuidado mais humano e efetivo. ${ }^{1}$ Dentre as linhas de cuidado, a gestação, em especial, é um período de grandes 
alterações fisiológicas e emocionais, durante o qual as ações educativas de prevenção e promoção da saúde devem ser priorizadas, e as necessidades assistenciais resguardadas, com vistas ao bem-estar do binômio mãe-filho. ${ }^{2,3}$

O pré-natal odontológico (PNO) consiste na assistência e controle da saúde bucal da gestante, no aconselhamento para a manutenção da saúde do bebê, objetivando o cuidado com os dentes e tecidos periodontais, reabilitação oral e correto desenvolvimento craniofacial da criança. ${ }^{4}$ Para a mulher, tem grande importância na medida em que previne e trata condições bucais relevantes ao período, tais como as infecções periodontais, fator que pode influenciar o parto prematuro e o baixo peso ao nascer. ${ }^{5}$ Para o bebê, o PNO justifica-se por ganhos futuros em termos de saúde bucal, a exemplo do correto desenvolvimento craniofacial e menor risco de cárie, alcançados através da intervenção em fatores de risco relacionado à infância, como o aleitamento materno, o uso de bicos artificiais e a exposição precoce ao açúcar. ${ }^{6}$

Sabe-se que a abordagem do curso de vida materno se apresenta como uma importante ferramenta na previsão e prevenção de patologias orais durante a primeira infância. Hábitos maternos, influenciados por suas condições de vida, tais como dieta, atividade física, obesidade e tabagismo são refletidos na formação das crianças, podendo exercer influências tanto positivas, quanto negativas. A exemplo disso, o desenvolvimento da doença cárie pode ser favorecido pelo estilo de vida da mãe durante a gestação, tal qual uma dieta rica em açúcares e gorduras, bem como o sobrepeso e obesidade. ${ }^{7}$ Nesse contexto, o PNO pode contribuir na abordagem e sensibilização das gestantes sobre a relação entre seu estilo de vida e a saúde bucal de seus filhos.

Porém, o PNO ainda não se apresenta como prática consolidada, e muitas vezes o contato com o cirurgião-dentista (CD) durante o período gestacional não acontece, ${ }^{8}$ o que faz da consulta pré-natal, realizada por médicos e enfermeiros, o único elo entre a gestante e os serviços de saúde. Em estudo realizado com 83 médicos ginecologistaobstetras em 2009, Araújo, Pohlmann e Reis ${ }^{9}$ demonstraram que apenas $51,8 \%$ destes 
profissionais aconselham suas pacientes a procurarem $O C D$ durante o pré-natal, caracterizando deficiência de ações multiprofissionais dentro do cuidado pré-natal. Além disso, ao avaliar dados do Programa de Melhoria do Acesso e Qualidade da Atenção Básica - PMAQ-AB de um município do estado de São Paulo, Moimaz e colaboradores ${ }^{10}$ evidenciaram baixa resolutividade dos serviços odontológicos e acesso prejudicado das gestantes aos mesmos, traduzidos pelos baixos índices de tratamentos concluídos e de primeiras consultas odontológicas programáticas.

A atuação de médicos e enfermeiros no cuidado pré-natal deve seguir as ações propostas pelo Ministério da Saúde, consubstanciadas em documentos como "Pacto pela Saúde" de 2006, ou no "Manual Técnico de Pré-Natal e Puerpério", editado em $2005{ }^{11}$ Estes documentos preveem a interdisciplinaridade intrínseca ao trabalho multiprofissional, incluindo ações de saúde bucal para gestantes mesmo durante o acompanhamento prénatal com médicos e enfermeiros. ${ }^{12}$ Atitudes e práticas de médicos e enfermeiros, tais como inspeção visual da cavidade oral, investigação sobre queixas bucais da gestante e o diálogo permanente com a equipe de saúde bucal sobre a condição bucal de gestantes acompanhadas, representam possibilidades de inserção destes profissionais pré-natal odontológico.

Neste contexto, é fundamental identificar como estes profissionais compreendem as questões de saúde bucal, suas relações com o período gestacional, e até que ponto tais conhecimentos são, efetivamente, convertidos em ações de cuidado. Assim, o objetivo do estudo foi descrever o conhecimento e práticas referentes à saúde bucal materno infantil, de profissionais da medicina e enfermagem envolvidos no cuidado pré-natal, na Atenção Básica à Saúde, da cidade de Vitória da Conquista, BA, em 2015.

\section{Metodologia}

Trata-se de um estudo transversal, censitário, com uma abordagem quantitativa e descritiva, que envolveu médicos e enfermeiros que atuavam em unidades de saúde, na atenção básica de Vitória da Conquista, BA, no ano de 2015. 
A cidade de Vitória da Conquista situa-se na região sudoeste da Bahia, e abriga uma população de, aproximadamente, 340.00 habitantes, o que faz do município a terceira maior cidade do estado baiano (IBGE, 2015). ${ }^{13}$ É a capital regional de uma área que abrange aproximadamente oitenta municípios na Bahia e dezesseis no norte de Minas Gerais. A Atenção Básica à Saúde de Vitória da Conquista conta com 38 Equipes de Saúde da Família - ESF, dentre as quais 15 estão situadas na zona urbana. Por outro lado, o município conta com 30 Equipes de Saúde Bucal - ESB, das quais sete encontram-se inseridas nas ESF urbanas, em sua maioria na proporção 1:2 (uma ESB para 2 ESF). Existem, ainda, sete Unidades Básicas de Saúde - UBS na zona urbana, as quais contém Equipe de Saúde Bucal.

A população de referência do presente estudo definiu-se por profissionais inseridos na Atenção Básica de Vitória de Conquista, BA, quer em Unidades Básicas de Saúde, quer em Unidades de Saúde da Família, em 2015. Para que a UBS fosse incluída no estudo, era necessário que a mesma contasse com Equipe de Saúde Bucal.

A inclusão dos indivíduos no estudo foi condicionada aos seguintes critérios: atuar na zona urbana de Vitória da Conquista, BA por, no mínimo, seis meses na unidade básica onde desenvolvia suas atividades profissionais. Além disso, era necessário que o profissional estivesse envolvido em ações de pré-natal em seu processo de trabalho. Por outro lado, foram excluídos do estudo os profissionais que se encontravam, por qualquer motivo, afastados de suas atividades no momento da coleta de dados.

Para a coleta dos dados, foi desenvolvido um questionário, composto por 54 questões, divididas em quatro blocos: aspectos socioeconômicos e demográficos dos participantes; expectativas/satisfação do profissional com o trabalho na atenção básica e cuidado materno infantil; conhecimentos dos profissionais quanto as possíveis relações entre gravidez e saúde bucal; e atitudes dos profissionais frente às questões de saúde bucal durante o cuidado pré-natal.

O questionário apresentava linguagem clara e objetiva, de forma a alcançar o 
entendimento dos sujeitos da pesquisa sobre cada variável proposta. A aplicação dos mesmos deu-se entre os meses de março e junho de 2015 , sob a forma de entrevista, sem que houvesse alteração da pergunta e/ou influência sobre a resposta do entrevistado pelo entrevistador. Para efetivação dessa fase da pesquisa, foi realizado o devido treinamento de 3 entrevistadores, a fim de dar suporte metodológico aos mesmos e evitar vieses na obtenção dos resultados.

Os resultados foram organizados em um banco de dados, a partir de digitação simples, utilizando o software Statistical Package for Social Sciences (SPSS) versão 22.0. Após isso, a análise realizada foi descritiva, e envolveu a obtenção de frequências absolutas e relativas, para as variáveis qualitativas, bem como medidas de tendência central e dispersão, para as variáveis quantitativas.

O trabalho foi conduzido dentro dos preceitos da bioética, presididos pela Resolução 466/12 do Conselho Nacional de Saúde, e foi aprovado pelo Comitê de Ética em Pesquisa da Faculdade Independente do Nordeste - FAINOR, processo CAAE: 38810514.8.0000.5578. Os profissionais que aceitaram participar do estudo foram informados em relação à finalidade e ao sigilo das informações coletadas e assinaram o Termo de Consentimento Livre e Esclarecido.

\section{Resultados}

O universo da pesquisa contava com 60 profissionais, sendo 21 médicos e 39 enfermeiros. Destes, três médicos se recusaram a participar do estudo; um médico encontrava-se em período de férias no momento da coleta. Ademais, dois enfermeiros não foram encontrados durante a abordagem dos entrevistadores. Desta forma, a população de estudo final foi composta por 54 profissionais, o que corresponde a $90 \%$ dos profissionais aptos à inclusão na pesquisa.

A população de estudo foi composta por 17 médicos (31,5\%) e 37 enfermeiros (68,5\%), a maioria inserida em Unidades de Saúde da Família (68,5\%), com média de 18 
meses de atuação, variando entre seis e 240 meses. Dentre os profissionais que declararam ter algum curso de pós-graduação $(70,4 \%)$, houve destaque para os cursos de Saúde da Família (16,7\%) e Saúde Pública (14,8\%) (Tabela 1).

Tabela 1. Caracterização profissional de médicos e enfermeiros inseridos na Atenção Básica de Vitória da Conquista, BA, Brasil. 2015

\begin{tabular}{lcc}
\multicolumn{1}{c}{ Variável } & n & $\%$ \\
Tipo de estabelecimento & 17 & 31,5 \\
UBS & 37 & 68,5 \\
USF & & \\
Profissão & 17 & 31,5 \\
$\quad$ Médico & 37 & 68,5 \\
Enfermeiro & & \\
Tempo de graduação & 16 & 29,6 \\
Até 3 anos & 13 & 24,1 \\
De 4-6 anos & 8 & 14,8 \\
De 7-9 anos & 3 & 5,6 \\
De 10-12 anos & 14 & 25,9 \\
De 13 ou mais & & \\
Tipo de instituição da graduação & 33 & 61,1 \\
Pública & 21 & 38,9 \\
Privada & & \\
Possui pós-graduação & 38 & 70,4 \\
Sim & 16 & 29,6 \\
Não &
\end{tabular}

Fonte: Elaboração própria (2015).

n=54; UBS - Unidade de Saúde Bucal; USF - Unidade de Saúde da Família

A maioria dos profissionais $(53,7 \%)$ relatou ter sido incentivada durante a graduação a se atentar à saúde bucal de gestantes.61,1\% não participaram de curso introdutório para atuação na Atenção Primária à Saúde - APS, e a maioria dos que participaram (66,7\%) relatou não haver abordagem sobre o cuidado em saúde bucal no pré-natal. Além disso, 74,1\% disseram já ter participado de estratégia educativa para o cuidado materno infantil, mas $50 \%$ destes apontaram que a saúde bucal não foi abordada. A maioria dos profissionais $(66,7 \%)$ apontou que os CDs já conduziram discussões com a equipe sobre a importância do pré-natal odontológico, porém 55,6\% declararam que os dentistas não enfatizaram aspectos de saúde bucal que devem ser valorizados durante a consulta pré- 
natal (Tabela 2).

Tabela 2. Formação e capacitação dos profissionais médicos/enfermeiros para o trabalho na Atenção Básica e cuidado materno-infantil de Vitória da Conquista, BA, Brasil. 2015

\begin{tabular}{|c|c|c|}
\hline Variável & $\mathbf{n}$ & $\%$ \\
\hline \multicolumn{3}{|c|}{ Abordagem na graduação sobre saúde bucal materno infantil $(n=54)$} \\
\hline Sim & 29 & 53,7 \\
\hline Não & 25 & 46,3 \\
\hline \multicolumn{3}{|c|}{ Fez curso introdutório para atuar na atenção básica $(n=54)$} \\
\hline Sim & 21 & 38,9 \\
\hline Não & 33 & 61,1 \\
\hline \multicolumn{3}{|l|}{ Avaliação do curso introdutório $(n=21)$} \\
\hline Satisfatório & 14 & 66,6 \\
\hline Pouco satisfatório & 6 & 28,6 \\
\hline Insatisfatório & 1 & 4,8 \\
\hline \multicolumn{3}{|c|}{ Abordagem da saúde bucal materno-infantil no curso introdutório $(n=21)$} \\
\hline Sim & 7 & 33,3 \\
\hline Não & 14 & 66,7 \\
\hline \multicolumn{3}{|c|}{ Participou de ações formativas para o cuidado materno infantil $(n=54)$} \\
\hline Sim & 40 & 74,1 \\
\hline Não & 14 & 25,9 \\
\hline \multicolumn{3}{|l|}{ Avaliação da ação formativa $(n=40)$} \\
\hline Satisfatória & 32 & 80 \\
\hline Pouco satisfatória & 7 & 17,5 \\
\hline Insatisfatória & 1 & 2,5 \\
\hline \multicolumn{3}{|c|}{ Abordagem da saúde bucal materno infantil na ação formativa $(n=40)$} \\
\hline Sim & 20 & 50 \\
\hline Não & 20 & 50 \\
\hline \multicolumn{3}{|c|}{ O CD apresenta à equipe a importância do pré-natal odontológico $(n=54)$} \\
\hline Sim & 36 & 66,7 \\
\hline Não & 18 & 33,3 \\
\hline \multicolumn{3}{|c|}{ O CD aponta condições bucais a serem observados na consulta da gestante $(n=54)$} \\
\hline Sim & 24 & 44,4 \\
\hline Não & 30 & 55,6 \\
\hline \multicolumn{3}{|c|}{ Autoavaliação do desempenho no cuidado materno-infantil $(n=54)$} \\
\hline Muito satisfatório & 13 & 24,1 \\
\hline Satisfatório & 41 & 75,9 \\
\hline Insatisfatório & 0 & 0 \\
\hline
\end{tabular}

Fonte: Elaboração própria (2015).

Os profissionais foram questionados sobre seus conhecimentos em saúde bucal materno infantil, e os resultados são apresentados na Tabela 3. A expressão "pré-natal odontológico" foi desconhecida por 33,3\% dos entrevistados. Ainda assim, a maioria 
$(96,3 \%)$ considerou o contato da gestante com o CD importante, independentemente de haver queixa bucal. Entre os que acreditaram que a gestante pode receber intervenção odontológica (98,1\%), uma grande parte (35,2\%) afirmou que os procedimentos cirúrgicos estão contraindicados a essas pacientes, e 59,3\% afirmaram não haver contraindicação absoluta a nenhum tratamento odontológico para gestantes. Ademais, $81,5 \%$ dos profissionais se julgaram muito seguros para o encaminhamento de gestantes ao $C D$, apesar de $66,0 \%$ avaliarem seus conhecimentos sobre saúde bucal como regular. Todos os profissionais apontaram uma relação bidirecional entre a gestação e a saúde bucal.

Tabela 3. Conhecimento de médicos e enfermeiros em saúde bucal da gestante. Atenção Básica de Vitória da Conquista, BA, Brasil. 2015

\begin{tabular}{|c|c|c|}
\hline Variável & $\mathbf{n}$ & $\%$ \\
\hline \multicolumn{3}{|l|}{ Conhece a expressão pré-natal odontológico $(n=54)$} \\
\hline Sim & 36 & 66,7 \\
\hline Não & 18 & 33,3 \\
\hline \multicolumn{3}{|l|}{ Opinião sobre o contato da gestante com o CD $(n=54)$} \\
\hline Importante independente de queixa bucal & 52 & 96,3 \\
\hline Importante quando houver queixa bucal & 2 & 3,7 \\
\hline \multicolumn{3}{|l|}{ A gestante pode receber intervenção odontológica $(n=54)$} \\
\hline Sim & 53 & 98,1 \\
\hline Não & 1 & 1,9 \\
\hline \multicolumn{3}{|l|}{ Que tipo de intervenção a gestante pode receber $(n=54)$} \\
\hline Qualquer tipo de intervenção & 32 & 59,2 \\
\hline Qualquer tipo de intervenção, exceto cirurgia & 19 & 35,2 \\
\hline Apenas prevenção e educação em saúde & 3 & 5,6 \\
\hline \multicolumn{3}{|c|}{ Segurança para encaminhar gestantes ao tratamento odontológico $(n=54)$} \\
\hline Muito seguro & 44 & 81,5 \\
\hline Pouco seguro & 8 & 14,8 \\
\hline Inseguro & 10 & 3,7 \\
\hline \multicolumn{3}{|l|}{ A gestante pode receber anestesia odontológica $(n=54)$} \\
\hline Sim & 48 & 88,9 \\
\hline Não & 6 & 11,1 \\
\hline \multicolumn{3}{|l|}{ A gestante pode realizar radiografia odontológica $(n=52)$} \\
\hline Sim & 22 & 42,3 \\
\hline Não & 30 & 57,7 \\
\hline \multicolumn{3}{|c|}{ O aleitamento materno pode influenciar a saúde bucal do bebê $(n=54)$} \\
\hline Sim & 51 & 94,4 \\
\hline Não & 3 & 5,6 \\
\hline
\end{tabular}




\begin{tabular}{|c|c|c|}
\hline Sim, de forma positiva & & 5,6 \\
\hline Sim, de forma negativa & 50 & 92,5 \\
\hline Não & 1 & 1,9 \\
\hline \multicolumn{3}{|l|}{ Quando começar a realizar higiene bucal do bebê $(n=54)$} \\
\hline Logo após o nascimento & 49 & 90,7 \\
\hline Quando iniciar a erupção dentária & 3 & 5,5 \\
\hline Quando finalizar a erupção dentária & 1 & 1,9 \\
\hline Quando iniciar o aleitamento materno complementado & 1 & 1,9 \\
\hline \multicolumn{3}{|l|}{ Uso de antibiótico aumenta o risco de cárie de crianças $(n=52)$} \\
\hline Sim & 29 & 55,8 \\
\hline Não & 23 & 44,2 \\
\hline \multicolumn{3}{|l|}{ Uso de xaropes interfere na saúde bucal de crianças $(n=53)$} \\
\hline Sim & 39 & 73,6 \\
\hline Não & 14 & 26,4 \\
\hline \multicolumn{3}{|l|}{ Como você julga seu conhecimento sobre saúde bucal } \\
\hline Suficiente & 11 & 20,8 \\
\hline Regular & 35 & 66 \\
\hline Insuficiente & 7 & 13,2 \\
\hline
\end{tabular}

Destaca-se que grande parte dos profissionais sustentou a contraindicação de tomada radiográfica odontológica em gestantes $(57,7 \%)$ e o aumento do risco de cárie em crianças pelo uso de antibióticos (55,8\%). Por outro lado, foi evidente o reconhecimento do efeito da gestação na saúde bucal da gestante $(98,1 \%)$, bem como do efeito do uso de xaropes $(73,6 \%)$, aleitamento materno $(94,4 \%)$ e bicos artificiais $(92,6 \%)$ na saúde bucal de crianças (Tabela 3).

A Tabela 4 apresenta os resultados sobre as ações em saúde bucal desenvolvidas por médicos/enfermeiros no cuidado pré-natal. A maioria dos profissionais afirmou questionar as gestantes sobre suas condições bucais $(44,4 \%$ sempre e $37,1 \%$ frequentemente), contudo, apontou nunca ou raramente realizara inspeção visual da cavidade oral da gestante durante a consulta pré-natal (61,1\%). O ausente (31,5\%) ou raro (25,9\%) diálogo entre médicos/enfermeiros e CDs sobre aspectos de saúde bucal (queixas, sinais e sintomas relatados) identificados durante a consulta pré-natal também foi evidenciado. Por fim, ações importantes para a saúde bucal durante o pré-natal foram relatadas pelos profissionais, tais como estímulo aos cuidados com a saúde bucal durante 
a gestação (37,0\% sempre e 35,2\% frequentemente); estímulo à realização de higiene oral do bebê (70,4\% sempre e 22,2\% frequentemente); e o incentivo ao aleitamento materno exclusivo até o $6^{\circ}$ mês de vida (100\% dos participantes).

Tabela 4. Atitudes e práticas em saúde bucal materno infantil de médicos e enfermeiros. Atenção Básica de Vitória da Conquista, BA, Brasil. 2015.

\begin{tabular}{|c|c|c|c|c|c|c|c|c|}
\hline \multirow[t]{2}{*}{ Prática $(n=54)$} & \multicolumn{2}{|c|}{$S$} & \multicolumn{2}{|c|}{$\mathbf{F}$} & \multicolumn{2}{|c|}{$\mathbf{R}$} & \multicolumn{2}{|c|}{$\mathbf{N}$} \\
\hline & n & $\%$ & $\mathbf{n}$ & $\%$ & n & $\%$ & n & $\%$ \\
\hline $\begin{array}{l}\text { Questiona a gestante sobre sua condição } \\
\text { bucal }\end{array}$ & 24 & 44,5 & 20 & 37 & 6 & 11 & 4 & 7,4 \\
\hline Realiza inspeção da boca da gestante & 11 & 20,4 & 10 & 18,5 & 15 & 28 & 18 & 33,3 \\
\hline Orienta a gestante a procurar o CD & 40 & 75,5 & 4 & 7,5 & 9 & 17 & 0 & 0 \\
\hline $\begin{array}{l}\text { Discute com o CD condições de saúde bucal } \\
\text { identificadas na consulta da gestante* }\end{array}$ & 12 & 22,2 & 11 & 20,4 & 14 & 26 & 17 & 31,5 \\
\hline $\begin{array}{l}\text { Estimula a gestante ao cuidado com a saúde } \\
\text { bucal }\end{array}$ & 20 & 37 & 19 & 35,2 & 9 & 17 & 6 & 11,1 \\
\hline Estimula a higiene oral do bebê & 38 & 70,4 & 12 & 22,2 & 4 & 7,4 & 0 & 0 \\
\hline $\begin{array}{l}\text { Orienta a higiene oral da criança após o uso } \\
\text { de xaropes** }\end{array}$ & 8 & 30,8 & 6 & 23,1 & 3 & 12 & 9 & 34,6 \\
\hline $\begin{array}{l}\text { Estimula o aleitamento materno após } \\
\text { introdução de alimentação }\end{array}$ & 51 & 94,4 & 3 & 5,6 & 0 & 0 & 0 & 0 \\
\hline $\begin{array}{l}\text { Orienta a gestante sobre o risco de bicos } \\
\text { artificiais }\end{array}$ & 39 & 72,2 & 10 & 18,5 & 3 & 5,6 & 2 & 3,7 \\
\hline $\begin{array}{l}\text { Orienta a gestante a retardar a introdução } \\
\text { de açúcar na dieta da criança }\end{array}$ & 41 & 75,9 & 10 & 18,5 & 2 & 3,7 & 1 & 1,9 \\
\hline Prescreve suplementação com flúor*** & 0 & 0 & 1 & 5,9 & 2 & 12 & 14 & 82,3 \\
\hline
\end{tabular}

Fonte: Elaboração própria (2015).

$\mathrm{S}=$ Sempre; $\mathrm{F}=$ Frequentemente; $\mathrm{R}=$ Raramente; $\mathrm{N}=$ Nunca; ${ }^{1} 1$ dado perdido; ${ }^{*} 28$ indivíduos não responderam

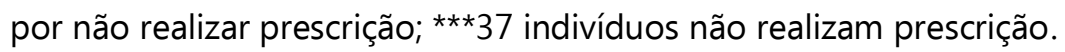

\section{Discussão}

Os achados deste estudo demonstraram que, apesar de existir consenso entre os médicos e enfermeiros sobre a importância do pré-natal odontológico, há fragilidades nos conhecimentos necessários para efetivação dessa prática, bem como dificuldades na efetivação de práticas centradas na abordagem multiprofissional. De maneira geral, os profissionais apresentaram bom desempenho nos conhecimentos mais gerais sobre o prénatal odontológico, como a importância do contato da gestante com o $C D$, o benefício do aleitamento materno para a saúde bucal do bebê, e o impacto da gestação na saúde bucal materna. Por outro lado, a investigação de conhecimentos mais específicos sobre o 
manejo odontológico na gestação revelou a existência de mitos, como a contraindicação de tomada radiográfica em gestantes ou a relação do antibiótico com o risco de cárie em crianças. Resultados semelhantes foram obtidos por George e colaboradores, em um estudo realizado com profissionais do pré-natal australiano em 2016, quando 53,9\% dos participantes apontaram sua falta de conhecimento sobre os riscos que envolvem o atendimento odontológico de grávidas, como uma barreira para suas ações voltadas ao pré-natal odontológico. ${ }^{14}$

Além disso, no âmbito das práticas referidas pelos profissionais esteve bem consolidado o encaminhamento para o CD e compartilhamento de informações de saúde bucal, mas houve deficiências na construção do cuidado compartilhado em saúde bucal, com vistas a integrá-lo como parte do complexo do pré-natal de forma dialógica e multiprofissional. A transferência da responsabilidade sobre a saúde bucal foi evidenciada pela não inclusão ou rara realização de inspeção visual da cavidade oral durante a consulta pré-natal. Além disso, o ausente ou raro diálogo dos médicos/enfermeiros com os CDs sobre aspectos de saúde bucal (queixas, sinais e sintomas) identificados durante a consulta pré-natal é um indicador da fragmentação do cuidado em saúde bucal na gestação. Os resultados vão ao encontro de outros estudos, que demonstraram a dificuldade, por parte dos profissionais envolvidos no pré-natal, em converter conhecimentos em ações que, de fato, favoreçam a saúde bucal de gestantes. ${ }^{15,16}$

Algumas características na população de estudo podem contribuir para a compreensão desse cenário, tais como a baixa participação dos profissionais em treinamentos introdutórios; a ausência de abordagem da saúde bucal nas ações formativas em saúde materno infantil; o curto tempo médio de atuação na atenção básica, que pode refletir a alta rotatividade profissional e fragilidade dos vínculos na equipe; a escassa comunicação dialógica entre os profissionais sobre as questões de saúde bucal; e a incipiente disponibilização de informações pelo dentista para embasamento da atuação de médicos e enfermeiros. Neste sentido, o fortalecimento do trabalho multiprofissional e da 
educação permanente mostra-se necessário para ampliação da participação de médicos e enfermeiros no cuidado em saúde bucal de gestantes e, consequentemente, para o fortalecimento do pré-natal odontológico.

O curso introdutório para profissionais ingressantes na atenção básica é uma importante ferramenta para alinhar a prática profissional às diretrizes do SUS e as linhas de cuidado em saúde. Apesar disso, Costa $^{17}$ verificou que muitas vezes nessas iniciativas há destaque exclusivo para políticas públicas, em detrimento da rotina de trabalho na atenção básica, as condutas e postura dos profissionais durante sua atuação, as possíveis dificuldades a serem encontradas, bem como seu enfrentamento.

O tempo médio de atuação dos profissionais no nível primário de atenção à saúde mostrou-se baixo, podendo ser um fator de quebra dos princípios do SUS. Sob a perspectiva do pré-natal odontológico, a alta rotatividade apresenta-se como entrave ao estabelecimento de relações de confiança, tanto entre os profissionais que compõem a equipe de saúde, quanto entre estes e a comunidade, uma vez que não há tempo hábil para a formação do vínculo e articulação de ações em saúde bucal para gestantes. Um dos grandes desafios da Estratégia de Saúde da Família é compor e manter equipes de trabalho que atendam aos preceitos do programa, tais como atuação generalista e formação de vínculo com a comunidade. Para Mendes, ${ }^{18}$ a alta rotatividade de médicos e enfermeiros da atenção básica pode estar relacionada à dinamicidade do mercado de trabalho, que muitas vezes oferece alternativas atrativas para atuação destes profissionais em outros espaços sociais.

As reuniões de equipe apresentam-se como importante estratégia para consolidação do trabalho multidisciplinar. Para Peduzzi, ${ }^{19}$ o trabalho na atenção básica deve ser desenvolvido sob os aspectos de equipe integração, na qual existe um projeto assistencial comum e autonomia técnica de caráter interdependente. Porém, os resultados desta pesquisa vão ao encontro de outros estudos, ${ }^{20,21}$ e apontam para uma atuação profissional pouco comunicativa, uma vez que os diálogos entre médicos/enfermeiros e 
CDs são raros, limitando o acesso dos primeiros a informações sobre saúde bucal de gestantes. Considerando que condições bucais, tais como doenças periodontais e experiência de cárie, podem influenciar o curso da gestação e a saúde bucal do bebê, a interação, o compartilhamento de saberes e um projeto assistencial alinhado entre os profissionais são fundamentais para que o trabalho desenvolvido durante o pré-natal seja, efetivamente, convertido em benefícios à gestante e ao bebê.

Muitos mitos ainda são sustentados por profissionais da enfermagem e medicina em relação à abordagem odontológica em gestantes, como por exemplo, a contraindicação de radiografias odontológicas e procedimentos cirúrgicos, e consequentemente, são transmitidos às pacientes. Em seu estudo, Codato et al. ${ }^{22}$ observaram que, em diversas situações, os profissionais podem contribuir com a manutenção de mitos entre as gestantes, uma vez que as mesmas depositam confiança inabalável em seus médicos, e os consideram como detentores do conhecimento sobre saúde geral, seguindo fielmente suas orientações. Muitas pacientes desconhecem a atuação generalista do CD, e acreditam que seus conhecimentos são limitados e restritos à cavidade oral, abstendo-se do acompanhamento odontológico por medo de injurias ao bebê e por acreditarem que as intervenções devem ser postergadas para o pós-parto. ${ }^{23} \mathrm{O}$ trabalho multiprofissional, valendo-se de recursos como agenda compartilhada e formação de grupos de gestantes com a participação conjunta de médicos, enfermeiros e CDs, apresenta-se como estratégia para o aumento da corresponsabilização e troca horizontal de informações, o que, por conseguinte, otimiza a consolidação do PNO e a assistência integral da gestante.

Os profissionais demonstraram reconhecer a importância dos cuidados odontológicos durante a gestação, apesar de terem recebido pouco incentivo durante a graduação e/ou participação em iniciativas para o cuidado materno-infantil. Contudo, o encaminhamento de gestantes para a consulta com o $C D$, quase sempre realizado por médicos e enfermeiros, pareceu ser uma transferência de responsabilidade, uma vez que a inspeção da cavidade oral e orientações pertinentes à saúde bucal não fazem parte da 
rotina das consultas de pré-natal. Venancio et al. ${ }^{12}$ relataram resultados semelhantes em seu estudo, quando enfermeiros demonstraram incerteza e insegurança sobre condições bucais relatadas por gestantes, procedendo o encaminhamento ao $C D$, mesmo quando a demanda se resumia em dúvidas e ações preventivas. A atuação dos profissionais envolvidos no cuidado pré-natal favorece o trabalho da ESB, na medida em que promove a sensibilização das pacientes quanto à necessidade de acompanhamento odontológico e seus benefícios para a unidade mãe-filho. Para que o PNO seja consolidado e inserido na atenção pré-natal, é necessário que o trabalho seja desenvolvido sob a perspectiva da transdisciplinaridade, ${ }^{24}$ no qual todos os profissionais de saúde são agentes sociais com olhares distintos sobre o mesmo objeto complexo, cada qual com seus paradigmas e axiomas que, somados de forma horizontal, convergem para o objetivo principal e comum: o cuidado integral às gestantes e bebês.

Apesar de demonstrarem conhecimento acerca do pré-natal odontológico, médicos e enfermeiros julgaram seus conhecimentos sobre saúde bucal como regulares, o que aponta a demanda por ações de capacitação, educação permanente e continuada em saúde bucal, de forma regular e sistemática. Para Andrade et al. ${ }^{25}$, a educação permanente, apesar de se apresentar como um desafio frente às particularidades políticas, sociais, culturais e ideológicas das ESF, gera resultados que justificam sua criação, aumentando o valor individual dos profissionais, sem perder de vista o trabalho multiprofissional e o objetivo comum.

Aponta-se com limitações desse estudo a construção de evidências através de dados autodeclarados e obtidos através de um instrumento não validado. Apesar do questionário utilizado não dispor de confiabilidade atestada por sua validação, o mesmo foi desenvolvido com embasamento científico sobre as diretrizes para saúde bucal de gestantes. Além disso, sugere-se novos estudos, tanto qualitativos quanto epidemiológicos, para melhor compreensão do tema e seus desdobramentos para a comunidade. 


\section{Conclusão}

O conhecimento apresentado por médicos e enfermeiros em relação à saúde bucal materno infantil esteve mais relacionado a aspectos gerais do pré-natal odontológico, que às questões norteadoras das práticas no processo de cuidado. Evidenciou-se a persistência de mitos como a contraindicação de procedimentos cirúrgicos e radiografias odontológicas para gestantes. Adicionalmente, em termos de práticas, observou-se o pouco envolvimento direto dos médicos e enfermeiros que frequentemente transferiram a responsabilidade exclusivamente ao dentista, o que foi bastante evidente no encaminhamento de gestantes sem a prévia inspeção oral, ou ainda na ausência de diálogo sobre as condições de saúde bucal das gestantes encaminhadas. Por fim, as práticas relatadas pelos profissionais foram basicamente educativas.

É preciso que médicos e enfermeiros sejam constantemente sensibilizados da importância do PNO, conhecendo as relações entre doenças orais e gestação, e assumindo seu papel de educadores também em saúde bucal. As práticas transdisciplinares e multiprofissionais têm valor incontestável para o desenvolvimento de uma linha de cuidado às gestantes, e devem ser alvos em iniciativas de capacitação e educação permanente. Sobretudo, é necessário que médicos e enfermeiros percebam que uma atuação generalista perpassa os cuidados restritos à profissão, devendo incluir um olhar holístico às pacientes e seu momento específico, e garantindo que mãe e filho estejam inseridos em uma rede de atenção integral.

\section{Referências}

1. Verdi MIM, Ros MA, Souza LRA. Saúde e sociedade. 2a ed. Florianópolis: UFSC; 2012.

2. Moreira MR, Santin GC, Matos LG, Gravina DBL. Pré-natal odontológico: noções de interesse. J Manag Prim Healh Care. 2015;6(1):77-85. https://doi.org/10.14295/jmphc.v6i1.234.

3. Peixoto CR, Freitas LV, Teles LMR, Campos FC, Paula PF, Damascendo AKC. O pré-natal 
na atenção primária: o ponto de partida para reorganização da assistência obstétrica. Rev Enferm UERJ. 2011;19(2):286-91.

4. Kloetzel MK, Huebner CE, Milgrom P. Referrals for dental care during pregnancy. J Midwifery Womens Health. 2011;56(2):110-7. https://doi.org/10.1111/j.15422011.2010.00022.x.

5. Vanterpoo ISF, Been JV, Houben ML, Nikkels PGJ, DeKrijger RR, Zimmermann LJI, et al. Porphyromonas gingivalis within Placental Villous Mesenchyme and Umbilical Cord stromaIs Associated with Adverse Pregnancy Outcome. PLoS ONE. 2016 Jan 5;11(1):e0146157. https://doi.org/10.1371/journal.pone.0146157.

6. Henry JA, Muthu MS, Swaminathan K, Kirubakaran R. Do oral health educational programmes for expectant mothers prevent early childhood caries? Systematic review. Oral Health Prev Dent. 2017;15(3):215-21. https://doi.org/10.3290/j.ohpd.a38522.

7. Wigen TI, Wang NJ. Maternal health and lifestyle and caries experience in preschool children. A longitudinal study from pregnancy to age 5 years. Eur J Oral Sci. 2011;119(6):463-8. https://doi.org/10.1111/j.1600-0722.2011.00862.x.

8. Pinheiro-Ferreira SMS, Silva JF, Silva RV, Pinheiro ES, Batista LD, Fernandes CG. Conhecimento em saúde bucal do bebê e expectativa relativa ao pré-natal odontológico: retrato de um município baiano de grande porte. Rev Fac Odontol Lins. 2015;25(2):19-30. http://dx.doi.org/10.15600/2238-1236/fol.v25n2p19-30.

9. Araújo SM, Pohlmann CS, Reis VG. Conhecimento e atitudes dos médicos ginecologistas/obstetras a respeito da saúde bucal da gestante. Rev Fac Odontol UPF. 2009;14(3):190-6. https://doi.org/10.5335/rfo.v14i3.808.

10. Moimaz SAS, Saliba TA, Arcieri RM, Lofego L, Saliba NA. Atenção à saúde maternoinfantil e saúde bucal nos ciclos do PMAQ-AB. J Manag Prim Healh Care. 2018; 9:e1. https://doi.org/10.14295/jmphc.v9i0.315.

11. Geniole LAI, Vieira CCA, Kodjaoglanian VL, Martins CCV. Assistência de enfermagem por ciclos de vida. Campo Grande (MS): Ed. UFMS, Fiocruz Unidade Cerrado Pantanal; 2011.

12. Venancio EQ, Venancio de Paula EMQ, Imada SR, Reis CB. A percepção do enfermeiro da saúde da família sobre saúde bucal na gestação. Cien Cuidado Saude. 2011;10(4):812-9. http://dx.doi.org/10.4025/cienccuidsaude.v10i4.18327.

13. Instituto Brasileiro de Geografia e Estatística (IGBE). População estimada: IBGE. Estimativas da população residente com data de referência 1o de julho de 2015 [citado 
em 7 ago. 2015]. Disponível em: https://cidades.ibge.gov.br/brasil/ba/vitoria-daonquista/panorama.

14. George A, Dahlen HG, Reat J, Ajwani S, Bhole S, Korda A, et al. What do antenatal care providers understand and do about oral health care during pregnancy: a cross-sectional survey in New South Wales, Australia. BMC Pregnancy Childbirth. 2016;16(1):382. https://dx.doi.org/10.1186\%2Fs12884-016-1163-x.

15. Govindasamy R, Narayanan M, Balaji VR, Dhanasekaran M, Balakrishnan K, Christopher A. Knowledge, awareness, and practice among gynecologists, medical practitioners and dentists in Madurai regarding association between periodontitis and pregnancy outcomes. J Indian Soc Periodontol. 2018;22(5): 447-50.

https://doi.org/10.4103/jisp.jisp_164_18.

16. Hashim R, Akbar M. Gynecologists' knowledge and attitudes regarding oral health and periodontal disease leading to adverse pregnancy outcomes. J Int Soc Prev Community Dent. 2014;4(Suppl 3):S166-72. https://doi.org/10.4103/2231-0762.149028.

17. Costa IS. Da mudança de modelo ao modelo de mudança: um estudo sobre o curso introdutório do PSF [dissertação]. Rio de Janeiro: Universidade do Estado do Rio de Janeiro; 2008.

18. Mendes CLA. Perfil do profissional médico na Estratégia de Saúde da Família no município do Rio de Janeiro: um modelo em transição [dissertação]. Rio de Janeiro: Escola Nacional de Saúde Pública Sergio Arouca; 2015.

19. Peduzzi M. Equipe multiprofissional de saúde: conceito e tipologia. Rev Saúde Pública. 2001;35(1):103-9. http://dx.doi.org/10.1590/S0034-89102001000100016.

20. Fernandes HN, Thofehrn MB, Porto AR, Amestoy SC, Jacondino MB, Soares MR. Interpersonal relationships in work of multiprofessional team of family health unit. Rev Pesqui Cuidado Fundam Online. 2015;7(1):1915-26. http://dx.doi.org/10.9789/21755361.2015.v7i1.1915-1926.

21. Medeiros CS, Carvalho RN, Cavalcanti PB, Salvador AR. O processo de (des)construção da multiprofissionalidade na Atenção Básica: limites e desafios a efetivação do trabalho em Equipe na Estratégia Saúde da Família Em João Pessoa-PB. Rev Bras Cienc Saude. 2011;15(3):319-28.

22. Codato LAB, Nakama L, Cordoni-Junior L, Higasi MS. Atenção odontológica à gestante: papel dos profissionais de saúde. Ciênc Saúde Colet. 2011;16(4):2297-301. 
http://dx.doi.org/10.1590/S1413-81232011000400029.

23. Catão CDS, Gomes TA, Rodrigues RQF. Evaluation of the knowledge of pregnant women about the relationship between oral diseases and pregnancy complications. Rev Odontol UNESP. 2015;44(1):59-65. http://dx.doi.org/10.1590/1807-2577.1078.

24. Almeida-Filho N. Transdisciplinaridade e saúde coletiva. Cien Saude Colet. 1997;2(12):5-20. http://dx.doi.org/10.1590/1413-812319972101702014.

25. Andrade RS, Caldas LBSN, Falcão MLP, Goes PSA. Processo de trabalho em unidade de saúde da família e a educação permanente. Trab Educ Saúde. 2016;4(2):505-21. http://dx.doi.org/10.1590/1981-7746-sip00108.

\section{Minicurrículo}

Rejane Marques Pereira | ORCiD: 0000-0001-8051-7648

Cirurgiã-dentista pela Faculdade Independente do Nordeste. Vitória da Conquista, BA, Brasil.

Suélem Maria Santana Pinheiro-Ferreira | ORCiD: 0000-0002-4470-3168

Doutora em Saúde Pública pela Universidade Federal da Bahia - UFBA (Área de concentração: Epidemiologia). Professora auxiliar da Área de Medicina, Departamento de Saúde, Universidade Estadual de Feira de Santana - UEFS. Feira de Santana, BA, Brasil.

Rogério Vieira Silva | ORCiD: 0000-0003-2967-4116

Mestre e Doutor em Odontologia pela Pontifícia Universidade Católica de Minas Gerais (Área de concentração: Endodontia). Professor da Graduação em Odontologia da Faculdade Independente do Nordeste. Vitória da Conquista, BA, Brasil.

Jinária Fernandes da Silva | ORCiD: 0000-0002-5400-4192

Cirurgiã-dentista pela Faculdade Independente do Nordeste. Vitória da Conquista, BA, Brasil.

Isis Cardoso Benício dos Santos | ORCiD: 0000-0001-7326-2587

Cirurgiã-dentista pela Faculdade Independente do Nordeste. Vitória da Conquista, BA, Brasil. 